\title{
The Research and Design of Remote Control Airship for Substation Inspection
}

\author{
Hongliang Wang \\ Postdoctoral Research Workstation of Yunnan Power \\ Grid Corporation \\ Kunming , China \\ e-mail: hongliang.whl@gmail.com
}

\author{
Min Cao ${ }^{2}$, Dada Wang ${ }^{2}$, Peng $\mathrm{Li}^{1}$, Shaoquan \\ Zhang $^{2}$, Erlei Tang ${ }^{3}$ \\ ${ }^{1}$ Postdoctoral Research Workstation of Yunnan Power \\ Grid Corporation \\ Kunming , China \\ ${ }^{2}$ YunNan Electric Power Test \&Research Institute \\ Group CO., LTD. Electric Power Research Institute \\ ${ }^{3}$ North China Electric Power University
}

\begin{abstract}
The main substation and transmission line inspection are using artificial inspection nowadays, especially under harsh operating environments and mountain difficult, the effect is not very satisfactory. But the number of helicopters is a limited ,about 1 2times every year. While the research and design of remote control aircraft based on DTMF technology will greatly solves these problems. This paper gives the system design, software systems, hardware systems and mechanical parts design, then introduces the codec in the remote system application methods to achieve substations and transmission lines for the purpose of inspection airship.
\end{abstract}

Keywords- Substation; DTMF; Remote C8051F226; Airship

\section{INTRODUCTION}

Substation is an important part of the power system. It uses advanced, reliable, integrated and environmentally smart devices, and to digital information of the station, a communication platform for networking, information sharing and standardization as the basic requirements. It can auto complete information collection, measurement, control, protection, metering and testing and other basic functions, and also support the grid with real-time automatic control, intelligent regulation, decision-making and collaborative interactive online analysis and other advanced features.

Remote control technology is based on the development of automatic control technology and communications technology. The current universal remote control system via infrared remote control transmitter and receiver chips, however, In this paper, based on mobile communication technology telephone communications and remote signaling DTMF decoder. At first we used MT8870 to determine the key signal, and combined C8051F320 microcontroller designed a Remote Control Airship.

\section{HARDWARE SYSTEM DESIGN}

\section{A. MCU System}

C8051F320 chip is fully integrated mixed-signal systemon-chip MCU with high-speed, flexible, low-cost characteristics, compatible with CIP-51 core. The speed of it is probably C- 51 series $10-15$ times. Added FLASH memory, with the ability to reprogram the system can be used for non- volatile data storage, and also allowing field upgrades of the 8051 firmware[1]. JTAG debug circuitry of chip allows the use of application systems installed in the final production MCU on non-invasive (does not occupy chip resources), fullspeed, in-system debugging. The debug system supports inspection and modification of memory and registers, breakpoints, observation point, single-step and run and halt commands. In this system, the master chip microcontroller primarily responsible for controlling the operation of the motor, the frequency signal's receiving and processing, and control voice recording, MT8870 decoding functions are implemented. [2].

\section{B. B. MT8870 System}

Dual Tone Multi Frequency (DTMF) is composed of high frequency and low frequency group, the high and low each contain four frequencies. A high frequency signal and a low frequency signal superimposed to form a combined signal, a total of 16 coding, could solve the rapid identification and processing signaling in a wireless communication system identification code, control, remote control system to solve the problem of instruction code. Besides DTMF integrated circuit also has a small, stable and reliable, anti-interference ability, low power consumption, large capacity, etc., it is widely used in wireless communications, remote control and other fields [3]. In this paper use the MT8870 complete dual-tone multi-frequency (DTMF) signal reception and identification. Decoder circuit of MT8870 is shown in Fig.1.

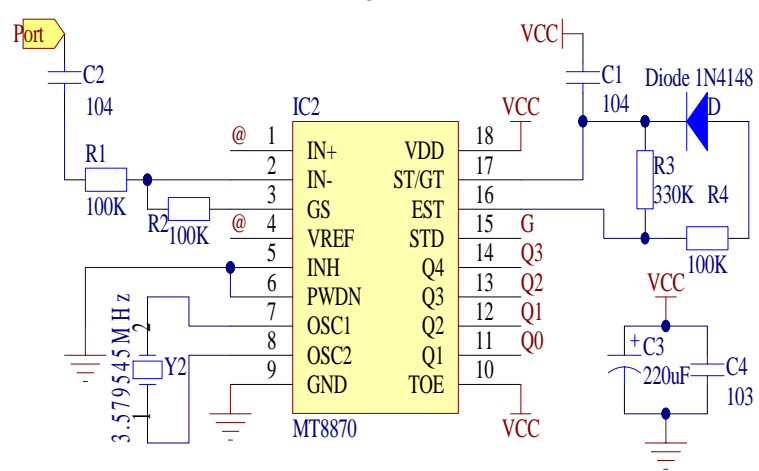

Fig.1. Decoder circuit of MT8870 


\section{Phonetic System}

In order to in the process of remote control airship can follow the voice prompts, and in the event of a fault alarm, especially to increase the voice system. ISD1420 phonetic system composed by a minimum application system, including a microphone, speakers, several resistive and capacitive components, two switches and power supplies. ISD1420 ChipCorder is a high-quality, short-cycle recording and playback circuits, use CMOS-technology, the internal contains on-chip clock, micro preamplifier, automatic gain control, band pass filter[4], smoothing filter, and power amplifier. ISD1420 phonetic system circuit is shown in Fig.2.

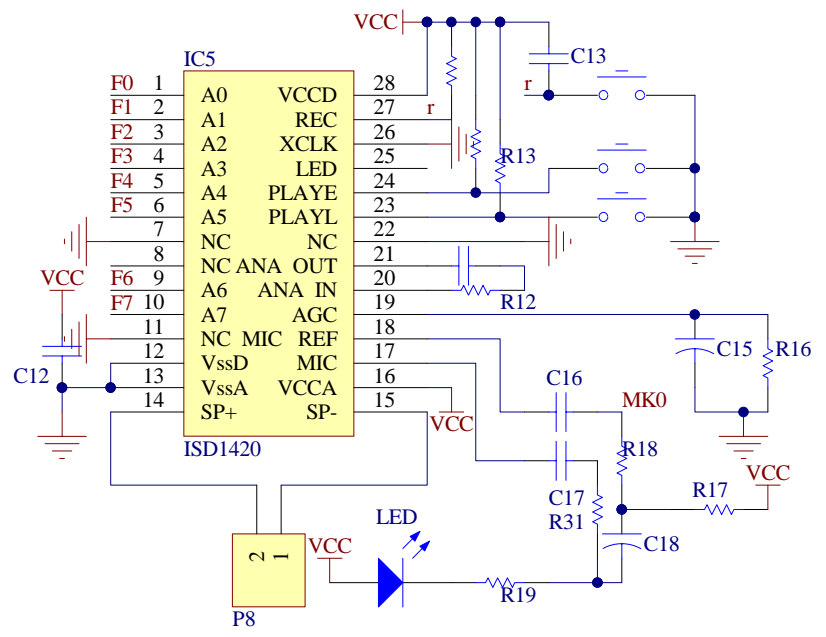

Fig.2. ISD1420 phonetic system circuit

\section{Substation System}

In Fig.3 shown that the architecture of substation inspection system based on airship.

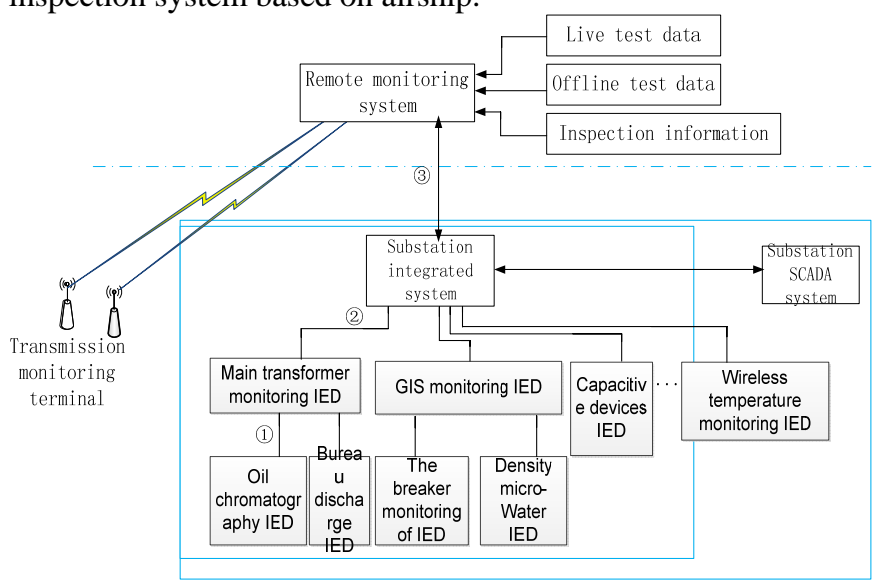

Fig.3. Substation system design architecture

In the substation create a master IED system, and establish a sub-IED systems for each monitoring project. Those IED establish a remote control system with the electricity sector layer or power network layer. The network layer adopt wireless sensor networks as priority network, power line carrier communication net, digital microwave network and other different communication networks as auxiliary network. To achieve perception layer and application layer the transmission of information and other functions, and provides high reliability, high security, largescale data transmission, in order to facilitate information sharing and interoperability, uniform application of IEC61850 protocol[5].

Sub-IED treatment online monitoring information from monitoring equipment in situ, such as pretreatment and early warning and forecast, then the results that after the information processing and analysis, fault information, early warning strategies and so on[6-8], to pass the station master IED. Master IED further analysis, processing, compared to data obtained from the sub-IED, then send strategies and results to remote monitoring systems and other substation IED system and obtain the necessary information and orders.

\section{RESEARCH OF SOFTWARE SYSTEM}

System main design flow is shown in Fig.4.

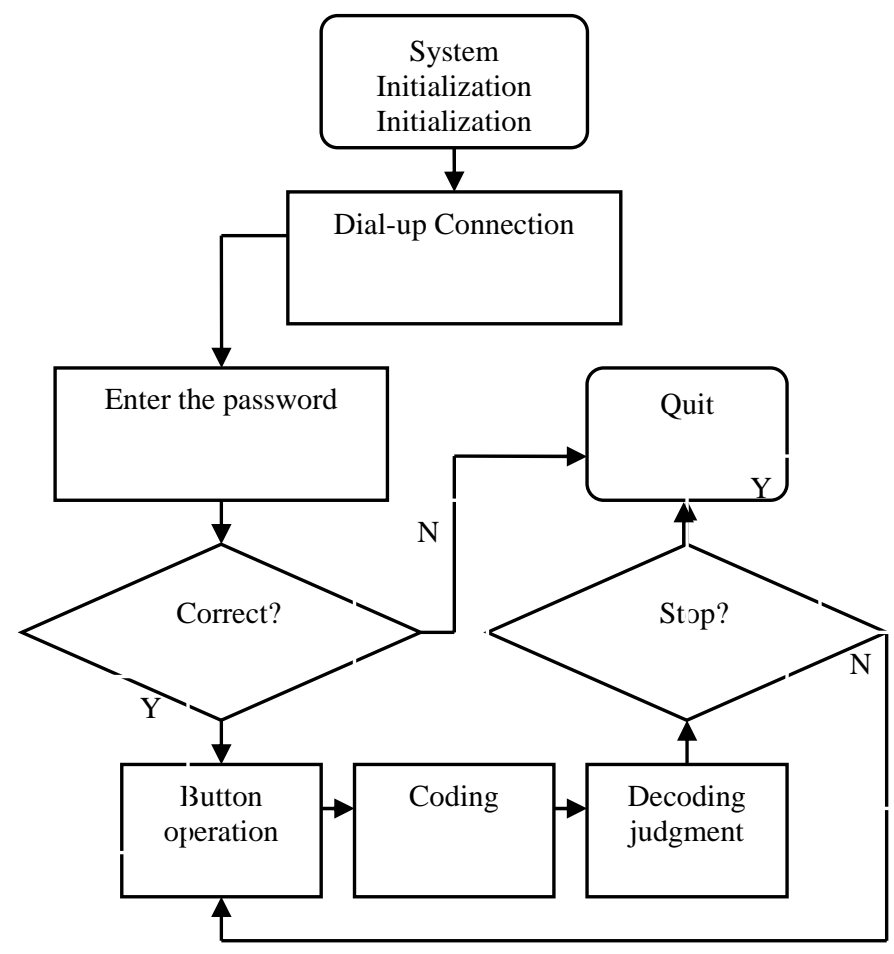

Fig.4. Main design flow

Partly of code are shown as follow:

\begin{tabular}{|c|c|c|}
\hline ST0: CLR & A & ; execute the main program \\
\hline SETB & $\mathrm{C}$ & \\
\hline MOV & R2,\#08 & ;R2 storing specific data \\
\hline ST1: RLC & A & \\
\hline MOV & $\mathrm{P} 2, \mathrm{~A}$ & \\
\hline ST2: RRC & A & \\
\hline $\begin{array}{l}\text { MOV } \\
\text { ACAL }\end{array}$ & $\begin{array}{l}\text { P2,A } \\
\text { DELAY }\end{array}$ & ; Microcontroller processing \\
\hline
\end{tabular}




\section{DJNZ R2,ST2 \\ AJMP ST0 \\ $\begin{array}{llll}\text { EXT0: } & \text { PUSH ACC } & \text { K } & \text { Key interrupt }\end{array}$ program \\ PUSH PSW \\ SETB RS0 \\ CLR RS1 \\ MOV P1,\#11110111B \\ ACALL DELAY2 \\ IV. THE DESIGN OF MECHANICAL PART}

In this design, the mechanical part comprises the actuator body, the actuator capsule, the auxiliary airbag, propulsion, pneumatic valves, exhaust nozzles, engine, catheters, radio receiver and the antenna, battery and so on. The design of system mechanical part is shown in Fig.6.

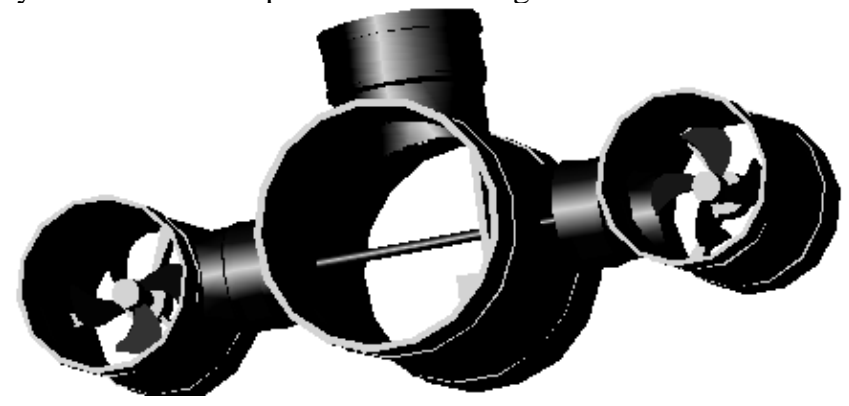

Fig.6. CAD drawing of the aircraft main body frame

\section{THE EXPERIMENTAL RESULTS}

The airship used the mathematical world classic honeycomb conjecture, using minimal materials to maximize use of space, reducing the weight of the aircraft itself. In addition, aircraft as a transportation of transport, their stability is very important. According planet in space, nature animals' eyes and limb sections, plants' dew, fruit and limb section are all exist as round and circular way, and only in this form of surface tension minimum, maximum stability, adapt to maximize the change of the nature of the harsh environment. Therefore, the airship shape are designed based on circle and arc, with rounded features and non-directional, the internal structure to rely on the principle of the honeycomb conjecture.[9-11]

In order to achieve the purpose of the flight, the use of mathematics in the relationship between point and surface, rational distribution its forward Motor System and balanced Motor System, and they are on the function both independent and cooperation[12]. They can achieve independently operation under artificial conditions. Their function are doubled under synergy operation, and also achieving the function of three-dimensional flight. According to physics principle of light and wave reflection, the outer surface of the airship is round and arc shape that will occurs diffuse light and wave. The light or the wave only on the vertical[13] point can return along the original direction, thereby increasing the airship hidden and practicality probing radar reflectivity. The Data of airship flight test are shown in Table I.
TABLE I.

THE EXPERIMENTAL DATA

\begin{tabular}{|l|l|}
\hline Airship length & $3 \mathrm{~m}$ \\
\hline Maximum flight speed & $12 \mathrm{~km} / \mathrm{h}$ \\
\hline Mean of flight altitude & $500 \mathrm{~m}$ \\
\hline Sustained flight time & $5 \mathrm{~h}$ \\
\hline Wind resistance & 3 level \\
\hline Course uniform error & $5^{\circ}$ \\
\hline Telemetry signal frequency & $1000 \mathrm{MHZ}$ \\
\hline Signal frequency & $75 \mathrm{MHZ}$ \\
\hline
\end{tabular}

\section{CONCLUSIONS}

In this paper, the design of the remote control airship from the status quo of substation and transmission line inspection, and DTMF-based technology. This paper introduces the codec on the remote system application methods, and gives the overall system design, software systems and mechanical design. Achieve the airship for the substation and transmission line inspection purposes. Flight test results show that the mission of airship designed can be completed for substation and transmission line inspection to provide a guarantee.

\section{ACKNOWLEDGMENT}

Paper is supported by the China Postdoctoral Science Foundation(2013M540721) and National High-Tech Research and Development Program(863) of China (2011AA05A120).

\section{REFERENCES}

[1] IEC61968 Application integration at electric utilities-system inter faces for distribution management[S].2009

[2] IEC 61970 Energy management system application program interface(EMS-API)[S].2004

[3] Wang Hongliang, Dong Haobin, Jiang Guosheng. Design of EMMWD signal detection system based on correlation and adaptive filter. Chinese Journal of Scientific Instrument, 2012 (05):1013 1018

[4] IEEE Std.802.15.42003.Wireless Medium Access Control(MAC) and Physical Layer(PHY) Specifications for Low Rate Wireless Personal Area Networks.2003.

[5] ZigBee Standards Organization. ZigBee Specification, Version07. ZigBee Alliance.2005.

[6] Wang Hongliang, Dong Haobin,etc. The Study of FID Signal Extraction Technology of Proton Magnetometer Based on SVD.International Journal of Digital Content Technology and its Applications ,2012.10(10):576 584

[7] Hongliang Wang, Haobin Dong. Study of the Distributed GroundTunnel Pressure Measurement and Control Method.The 2010 International Conference on Mechanical and Aerospace Engineering, KL,2010,6(6):48 51

[8] Guan, X., Y. Fei and H. Lin. Hierarchical Design of an ApplicationSpecific Instruction Set Processor for High-Throughput and Scalable FFT Processing. IEEE Transactions on Very Large Scale Integration Systems, 2011,5(99): 1 13

[9] Wang Hongliang,Dong Haobin,He Lianghua,Shi yongle, Zhang Yuan. Design and simulation of LQR controller with the linear 
inverted pendulum. International Conference on Electrical and Control Engineering, ICECE 2010,6: 699 702

[10] Hongliang Wang, Haobin Dong, Study of the Distributed GroundTunnel Pressure Measurement and Control Method[C]//The 2010 International Conference on Mechanical and Aerospace Engineering, KL,2010:48-51.
[11] Hongliang Wang, Haobin Dong, Design and Simulation of LQR Controller with the Linear Inverted Pendulum,ICECE 2010,699-702.

[12] Nianxi Xue, Signal Processing Applications with MATLAB(2nd edition), beijing:tsinghua university press,2008.1.

[13] Hao Jian, et al, "Moisture effect on the dielectric response and space chargeavior of mineral oil impregnated paper insulation", Dielectrics, university of Kent, UK, pp. 13-15, April, 2011. 